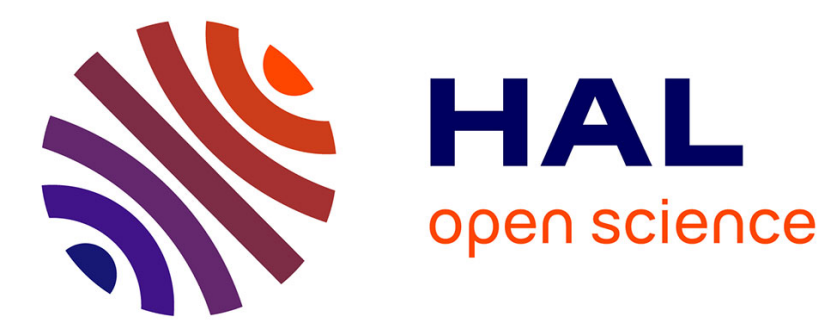

\title{
Continuum mechanics studies of plastic instabilities
}

\author{
A. Needleman
}

\section{To cite this version:}

A. Needleman. Continuum mechanics studies of plastic instabilities. Revue de Physique Appliquée, 1988, 23 (4), pp.585-593. 10.1051/rphysap:01988002304058500 . jpa-00245806

\section{HAL Id: jpa-00245806 https://hal.science/jpa-00245806}

Submitted on 1 Jan 1988

HAL is a multi-disciplinary open access archive for the deposit and dissemination of scientific research documents, whether they are published or not. The documents may come from teaching and research institutions in France or abroad, or from public or private research centers.
L'archive ouverte pluridisciplinaire HAL, est destinée au dépôt et à la diffusion de documents scientifiques de niveau recherche, publiés ou non, émanant des établissements d'enseignement et de recherche français ou étrangers, des laboratoires publics ou privés. 


\title{
Continuum mechanics studies of plastic instabilities
}

\author{
A. Needleman
}

Division of Engineering, Brown University, Box D, Providence RI 02912, U.S.A.

\author{
(Reçu le 15 juin 1987, accepté le 7 septembre 1987)
}

\begin{abstract}
Résumé.- Le formalisme de la mécanique des milieux continus est discuté pour l'analyse des instabilités par striction et par bandes. L'apparition de l'instabilité que l'on prédit dépend fortement des caractéristiques des matériaux, pas seulement à travers les propriétés comme le durcissement par écrouissage ou la sensibilité à la vitesse que l'on peut mesurer dans des tests conventionnels, mais aussi à travers la réponse des matériaux aux chemins de chargement. Un problème spécifique est discuté qui illustre l'influence de la courbure des surfaces d'écoulement, même quand un mécanisme d'adoucissement domine l'établissement de la localisation.
\end{abstract}

\begin{abstract}
The continuum mechanics framework for analyzing necking and shear band instabilities is discussed. The predicted onset of instability depends sensitively on the material's constitutive characterization, not only through properties, such as strain hardening and strain rate sensitivity, that can be measured in proportional loading tests, but also through the material's response to a change in loading path. A specific problem is discussed that illustrates the influence of the curvature of flow potential surfaces even when a softening mechanism plays a major role in precipitating localization.
\end{abstract}

\section{Introduction}

A broad range of behaviors can be regarded as instances of "plastic instability" or of "plastic flow localization." Examples include jerky flow, Lüders bands, necking of tensile specimens and shear bands. The class of plastic instability phenomena considered here are those where a macroscopically homogeneous or smoothly varying pattern of plastic deformation develops at low strains and then at larger strains gives way, more or less abruptly, to a highly localized deformation pattern. Such plastic instabilities can be categorized as either geometric or material. Geometric instabilities are those where features of the geometry of the body, such as a free surface, permit the instability mode to emerge and are the "material's" analog of the buckling instabilities encountered in structural mechanics. By way of contrast a material instability is insensitive to boundary conditions and can arise when the boundary constraints rule out geometric instabilities.

In ductile metals, the main manifestation of a material instability is a shear band. Often the large localized strains in a shear band precipitate a shear fracture. In other circumstances shear bands do not lead to fracture but localized shearing becomes an important mechanism for subsequent plastic deformation. Hence shear bands have a dual significance; as a precursor to fracture and as a mechanism of large strain plastic response.

The physical mechanisms responsible for triggering shear bands can vary widely. In some circumstances it appears that localization is an inherent and perhaps, as Asaro [1] has remarked, inevitable consequence of the plastic flow process. In such circumstances, a key feature of plastic material response for local- ization is the yield surface vertex structure implied by the discrete nature of crystallographic slip. In the present context, the significance of a vertex lies in the reduced stiffness to a change in loading path which permits shear bands to emerge in strain hardening materials.

A variety of softening mechanisms can act to promote localization. For example, lattice reorientation during deformation may lead to textures that are "soft" with respect to localized shearing, Dillamore et al. [2], Chang and Asaro [3] and Morii et al. [4]. At high rates of loading thermally induced softening due to local heating can lead to shear band development, Zener and Hollomon [5], Rogers [6] and Costin et al. [7]. The same metal may also undergo shear localization at very low strain rates, where thermal effects are negligible, with, for example, the softening due to progressive micro-rupture inducing localization.

Thus, depending on circumstances, localization can emerge either as a consequence of the material's plastic flow properties or as the result of an explicit softening process. Within the past decade or so, much attention has been given to the mechanics of plastic flow localization. Overviews of mechanics issues in localization can be found in Rice [8], Needleman and Rice [9], Peirce et al. [10], Needleman and Tvergaard [11], Asaro [12] and Tvergaard [13]. Here, the continuum framework for analyzing necking and shear band instabilities is outlined, but attention is primarily focussed on a single problem with the aim of illustrating the significance of the material's multi-axial constitutive description even in the presence of softening. The problem discussed is the analysis of plane strain compression in a thermally softening solid carried out by LeMonds and Needleman [14]. 


\section{Field Equations}

In a Lagrangian formulation the position of each material particle is labelled in a conveniently chosen reference configuration and these labels, together with time, serve as the set of independent variables. The labels can be identified with the position of the given particle relative to the origin of a fixed Cartesian frame and this position will be denoted by $\mathbf{x}$. In order to establish notation and to define field quantities, a brief outline of this formulation of the field equations will be given. Dyadic notation will be used and for the present purposes, it will not be necessary either to specify a reference configuration or to develop the equations in component form. Of course, to solve any specific boundary value problem a commitment needs to be made to some reference configuration and to a specific coordinate frame.

In the current configuration the material point initially at $\mathbf{x}$ is at $\overline{\mathbf{x}}$. The displacement vector $\mathbf{u}$ and the deformation gradient $F$ are defined by

$$
\mathbf{u}=\overline{\mathbf{x}}-\mathbf{x} \quad \mathbf{F}=\frac{\partial \overline{\mathbf{x}}}{\partial \mathbf{x}}
$$

The rate of deformation tensor is defined by

$$
\mathbf{D}=\frac{1}{2}\left(\dot{\mathbf{F}} \cdot \mathbf{F}^{-1}+\mathbf{F}^{-T} \cdot \dot{\mathbf{F}}^{T}\right)
$$

where ()$^{-T}$ is the inverse transpose and $\left(^{\circ}\right)$ is $\partial() / \partial t$.

Equilibrium can be expressed either in terms of the symmetric Cauchy stress tensor $\boldsymbol{\sigma}$ or the nonsymmetric nominal stress tensor s. These are related to the force, df, transmitted across a material element by

$$
\mathbf{d f}=\overline{\mathbf{n}} \cdot \boldsymbol{\sigma} d \bar{S}=\mathbf{n} \cdot \mathbf{s} d S
$$

where $d \bar{S}$ and $\overline{\mathbf{n}}$ give the area and orientation, in the current configuration, of a material element that had area $d S$ and orientation $\mathbf{n}$ in the reference configuration.

It is convenient to express the plastic flow rule in terms of the Kirchhoff stress defined by

$$
\boldsymbol{\tau}=\operatorname{det}(\mathbf{F}) \boldsymbol{\sigma}
$$

where $\operatorname{det}(\mathbf{F})$ is the ratio of the volume of a material element in the current configuration to its volume in the reference configuration. When plastic flow is volume preserving and when stress levels remain small compared to elastic moduli, then $\operatorname{det}(\mathbf{F}) \approx 1$ (presuming the reference configuration is some actual configuration of the body) and there is little difference between the Kirchhoff stress $\boldsymbol{\tau}$ and the Cauchy or true stress $\sigma$.

In the absence of body forces, rate equilibrium is expressed by

$$
\nabla \cdot \dot{\mathbf{s}}=0
$$

where $\left(^{\circ}\right)$ is $\partial() / \partial t$ and $\nabla$ denotes the gradient operator in the reference frame.

For the class of inelastic materials considered here, the constitutive relation is expressed as (see (3.8) and (3.9) to follow)

$$
\dot{\mathbf{s}}=\mathbf{K}: \dot{\mathbf{F}}-\dot{\mathbf{Q}}
$$

In the simplest case the boundary conditions take the form

$$
\left\{\begin{array}{l}
\mathbf{n} \cdot \dot{\mathbf{s}}=f \text { on } S_{t} \\
\dot{\mathbf{u}}=g \text { on } S_{u}
\end{array}\right.
$$

where $S_{t}$ is that part of the boundary on which tractions are prescribed and $S_{u}$ is that part of the boundary on which displacements are prescribed.

When the heating due to plastic dissipation is accounted for, balance of energy is written as

$$
\rho c_{p} \frac{\partial T}{\partial t}=\nabla \cdot(k \nabla T)+\chi \boldsymbol{T}: \mathbf{D}^{p}
$$

where $T$ is the temperature, $\rho$ is the density, $c_{p}$ is the heat capacity, $k$ is the thermal conductivity and the parameter $\chi$ specifies the fraction of plastic stress working converted to heat, which is typically in the range 0.85 to 0.95 , Taylor and Quinney [15].

\section{Constitutive Relations}

Attention is confined to circumstances where the elastic strains remain small, but the plastic strains can be large. The total rate of deformation, $\mathbf{D}$, is written as the sum of an elastic part, $\mathbf{D}^{\mathbf{e}}$, and a plastic part $\mathbf{D}^{\mathbf{p}}$ so that

$$
\begin{gathered}
\mathbf{D}=\mathbf{D}^{\mathbf{e}}+\mathbf{D}^{\mathbf{P}} \\
\mathbf{D}^{\mathbf{e}}=\frac{1+\nu}{E} \hat{\boldsymbol{\tau}}-\frac{\nu}{E}(\hat{\boldsymbol{\tau}}: \mathbf{I}) \mathbf{I}
\end{gathered}
$$

where $\hat{\boldsymbol{\tau}}$ is the Jaumann rate of Kirchhoff stress, $I$ is the identity tensor, $E$ is Young's modulus and $\nu$ is Poisson's ratio. The relation (3.2) is a consistent approximation to a hyperelastic relation when the elastic response is isotropic and when stress levels remain small compared to elastic moduli.

Plastic instability predictions are quite sensitive to the nature of the flow rule for $D^{p}$. The most widely used multi-axial plastic constitutive description is that of an isotropically hardening Mises solid, for which the flow rule takes the form

$$
\mathrm{D}^{\mathbf{p}}=\frac{3 \dot{\bar{\epsilon}}}{2 \bar{\sigma}} \boldsymbol{\tau}^{\prime}
$$

Here, $\dot{\bar{\epsilon}}$ is the effective plastic strain and $\bar{\sigma}$ is defined by

$$
\boldsymbol{\tau}^{\prime}=\boldsymbol{\tau}-\frac{1}{3}(\boldsymbol{\tau}: \mathrm{I}) \mathbf{I} \quad \bar{\sigma}^{2}=\frac{3}{2} \boldsymbol{\tau}^{\prime}: \boldsymbol{\tau}^{\prime}
$$

For a rate independent strain hardening solid

$$
\dot{\bar{\epsilon}}= \begin{cases}\left(\frac{1}{E_{\imath}}-\frac{1}{E}\right) \dot{\bar{\sigma}} \text { for } \bar{\sigma}=Y_{\max } \text { and } \dot{\bar{\sigma}}>0 \\ 0 & \text { for } \bar{\sigma}<Y_{\max } \text { or } \dot{\bar{\sigma}}<0\end{cases}
$$

where $Y$ is the current flow strength, which is the greater of the initial yield strength and the maximum value of $\bar{\sigma}$ attained over the deformation history and $E_{t}$ is the slope of the uniaxial Kirchhoff stress- logarithmic strain curve.

For a rate dependent solid (3.3) still holds but (3.5) is replaced by

$$
\dot{\bar{\epsilon}}=\dot{\epsilon}_{0}[\bar{\sigma} / g(\bar{\epsilon})]^{1 / m}
$$

The function $g(\bar{\epsilon})$, with $\bar{\epsilon}=\int \dot{\bar{\epsilon}} d t$, represents the effective stress versus effective strain response in a tensile test carried out at a strain-rate such that $\dot{\bar{\epsilon}}=\dot{\epsilon}_{0}$ and $m$ is the strain rate hardening exponent. For example, the function $g(\bar{\epsilon})$ can have the simple power law form

$$
g(\bar{\epsilon})=\sigma_{0}\left(\bar{\epsilon} / \epsilon_{0}+1\right)^{N} \quad \epsilon_{0}=\sigma_{0} / E
$$


where $\sigma_{0}$ is a reference strength and $N$ is the strain hardening exponent.

In the present context, the fundamental distinction between rate dependent material behavior as embodied in (3.6) and the rate independent behavior in (3.3), is that the plastic strain rate in (3.6) does not depend on incremental quantities. This leads to fundamentally different types of stress rate-strain rate relations for rate independent and rate dependent solids. In either case, the stress rate-strain rate relation is obtained by substituting (3.5) or (3.6) into (3.3), combining with (3.1) and (3.2) and inverting to obtain a relation between the Jaumann derivative of Kirchhoff stress and the rate of deformation tensor. Standard kinematic relations then convert this into a relation of the form (2.6) between nominal stress rate and deformation gradient rate.

For a rate independent solid, $\dot{\mathbf{Q}}=0$ and

$$
\dot{\mathbf{s}}= \begin{cases}\mathbf{K}_{\text {tan }}: \dot{\mathbf{F}} & \text { for plastic loading } \\ \mathbf{K}_{\text {elastic }}: \dot{\mathbf{F}} & \text { for elastic unloading }\end{cases}
$$

The relation (3.5) gives rise to a tensor of moduli that depends on incremental quantities through the loading-unloading condition. By way of contrast, for the rate dependent solid

$$
\dot{\mathbf{s}}=\mathbf{K}_{\text {elastic }}: \dot{\mathbf{F}}-\dot{\mathbf{Q}}
$$

The plasticity is embodied in $\dot{\mathbf{Q}}$ and, furthermore, $\dot{\mathbf{Q}}$ is independent of incremental field quantities as, of course, are the elastic moduli. There is no explicit loading-unloading condition for the rate dependent solid.

For values of the rate hardening exponent representative of structural metals at room temperature, say $m$ between 0.002 and 0.02 , the uniaxial response of the rate dependent solid is much like that of the corresponding rate independent solid. This is shown in Fig. 1 where the small strain uniaxial response is plotted.

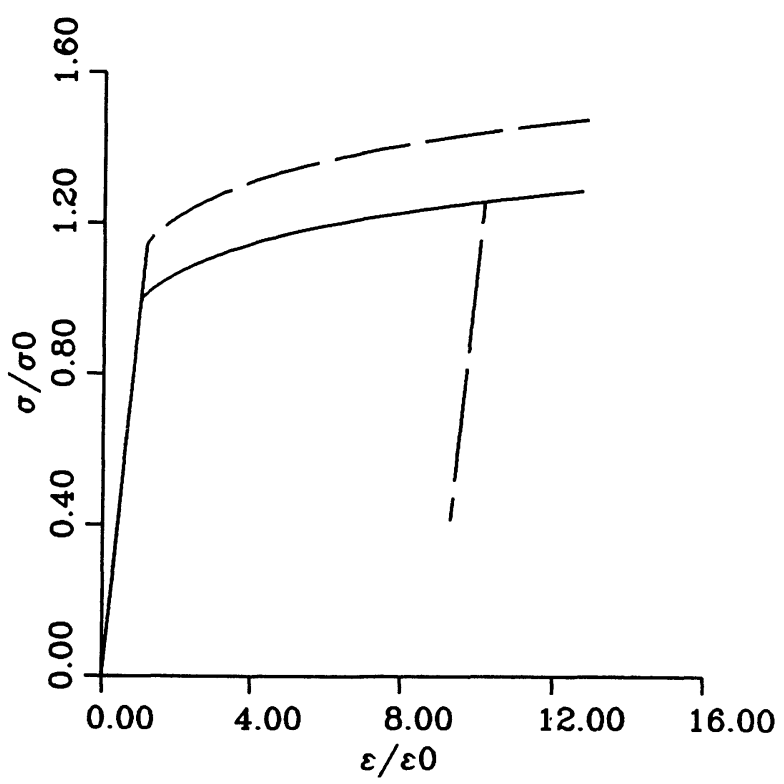

Figure-1 Stress-strain curves in uniaxial tension for a rate dependent solid with $m=0.01$. $-\dot{\epsilon} / \dot{\epsilon}_{0}=1 ;--\dot{\epsilon} / \dot{\epsilon}_{0}=10^{6}$; - - the response under strain rate reversal.

The relation (3.6) gives rise to an apparent rate dependent yield strength and to "unloading" like response on strain rate rever- sal, even though explicit yielding is not incorporated into the constitutive description.

Basic physical assumptions embodied in the flow rule are that the plastic strain rate has a direction normal to the flow potential surface in stress space, which for rate independent solids coincides with the yield surface. Furthermore, this surface is smooth, with a unique normal at the current stress point. Also, the plastic strain rate is volume preserving and pressure insensitive.

In rate independent plasticity, one consequence of a smooth yield surface is that the plastic strain rate direction is not influenced by the stress rate. This is at variance with predictions of physical plasticity models for polycrystalline aggregates based on the concept of single crystal slip. Such models inevitably lead to the development of a yield surface corner at the current loading point, Hill [16], Hutchinson [17], with the plastic strain rate direction depending, within limits, on the stress rate. Direct experimental evidence for corners is conflicting, although in some cases there is evidence for a region of high curvature at the current loading point, Hecker [18]. Recent analyses by Pan and Rice [19] and Asaro and Needleman [20] show that slight material rate sensitivity can account for the experimental ambiguity.

The main significance of a yield surface vertex for localization phenomena lies in the softer response to an abrupt change of loading path that occurs when the plastic strain rate can follow the stress rate. The $J_{2}$ corner theory of Christoffersen and Hutchinson [21] is an analytically tractable phenomenological theory of plasticity that incorporates key features exhibited by physical models of polycrystalline aggregates. The yield surface in the neighborhood of the loading point is taken to be a cone in stress deviator space. The derivation of the $J_{2}$ corner theory flow rule is complex and is described in Christoffersen and Hutchinson [21] and Hutchinson and Tvergaard [22].

For rate dependent solids a flow potential vertex is ruled out on quite general grounds, Rice [23]. In the investigations to be discussed here, a kinematic hardening flow rule is used to model a "rounded vertex." This use of kinematic hardening theory is intended for loading paths that do not involve extreme deviations from proportional loading and is quite distinct in focus from the use of kinematic hardening as a model for Bauschinger effects. The rate dependent polycrystal model of Asaro and Needleman [20] does exhibit a rounded vertex on suitably defined subsequent yield surfaces. Although, kinematic hardening is a rather limited model of a rounded vertex it does permit the interaction of vertex like behavior with softening mechanisms to be explored. Furthermore, within the context of rate independent plasticity, Tvergaard [24] and Hutchinson and Tvergaard [25] have shown that a solid having a smooth yield surface but with a high curvature (relative to the isotropic hardening surface) at the current loading point can give predictions of necking and shear band instabilities in line with those based on a corner theory of plasticity.

A simple combined isotropic-kinematic hardening material description is used by LeMonds and Needleman [14] with flow potential surfaces taken to be concentric cylinders about a stress state $\boldsymbol{\alpha}$. Defining

$$
\overline{\boldsymbol{\tau}}^{\prime}=\boldsymbol{\tau}^{\prime}-\alpha
$$

and

$$
\sigma_{F}^{2}=\frac{3}{2} \overline{\boldsymbol{\tau}}^{\prime}: \overline{\boldsymbol{\tau}}^{\prime}
$$

The flow rule (3.3) is replaced by 


$$
\mathrm{D}^{\mathbf{p}}=\frac{3 \dot{\bar{\epsilon}}}{2 \sigma_{F}} \overline{\boldsymbol{\tau}}^{\prime}
$$

with

$$
\dot{\bar{\epsilon}}=\dot{\epsilon}_{0}\left[\frac{\sigma_{F}}{\left[1-\beta\left(T-T_{0}\right)\right] h(\bar{\epsilon})}\right]^{1 / m}
$$

and

$$
h(\bar{\epsilon})=b \sigma_{0}\left[1+\epsilon / \epsilon_{0}\right]^{N}+(1-b) \sigma_{0}
$$

where $T_{0}$ is a reference temperature and $\beta$ specifies the thermal softening characteristics of the material.

The center of the flow potential surfaces evolves according to

$$
\hat{\boldsymbol{\alpha}}=\frac{2}{3}(1-b) \rho \mathrm{D}^{p}
$$

where

$$
\rho=N\left(\dot{\bar{\epsilon}} / \dot{\epsilon}_{0}\right)^{m}\left[1-\beta\left(T-T_{0}\right)\right] \frac{\sigma_{0}}{\epsilon_{0}}\left(\bar{\epsilon} / \epsilon_{0}+1\right)^{N-1}
$$

The parameter $b$ varies between zero and unity; $b=1$ corresponds to isotropic hardening and $b=0$ corresponds to kinematic hardening. Values between these two extremes correspond to a combination of isotropic expansion and translation of surfaces of constant flow potential.

The flow rule (3.12) is combined with (3.2) and gives a stress rate-deformation gradient rate relation of the form (3.9). In the formulation of LeMonds and Needleman [14] temperature dependence of plastic flow is incorporated into the analysis via (3.13) and (3.16), but thermo-elastic effects are not accounted for.

\section{Plastic Instabilities}

Here by a plastic instability is meant the more or less abrupt change from one deformation pattern to another. Examples include necking of tensile bars and shear band localizations. A general theory of plastic instabilities is only available for quasistatically and isothermally deforming rate independent solids and, furthermore, when the rate constitutive equations have a potential structure. Hill's $[26,27]$ theory of bifurcation is then available to determine the critical strain at which a deformation pattern ceases to be the unique solution for a given boundary value problem. Even in this context, a precise connection between loss of uniqueness and loss of stability in the dynamic sense remains to be developed, IIill [27]. The theory is much less well developed when such a potential structure is not present, although steps toward the development of a bifurcation theory for such solids have been taken, Raniecki and Bruhns [28].

\subsection{Geometric Instabilities}

For the standard boundary problem, (2.5)-(2.7), suppose that, at a given stage of the deformation history two solutions are possible. Denote the difference between field quantities associated with the two solutions by $\Delta\left({ }^{\circ}\right)$, so that if both incremental fields are solutions to the rate boundary value problem, the $\Delta\left({ }^{\circ}\right)$ fields satisfy

$$
\nabla \cdot \Delta \dot{\mathbf{s}}=0
$$

together with

$$
\Delta \dot{\mathbf{u}}=0 \text { on } S_{u} \quad \mathbf{n} \cdot \Delta \dot{\mathbf{s}}=0 \text { on } S_{t}
$$

For rate independent solids, when the constitutive relation admits a potential structure, Hill's $[26,27]$ bifurcation theory shows that the plastic loading branch of (3.8) is to be used in relating $\Delta \dot{\mathbf{s}}$ and $\Delta \dot{\mathbf{F}}$ throughout the current plastic zone. This, in conjunction with (4.1) and (4.2) leads to an eigenvalue problem governing the onset of bifurcation.

Hill and Hutchinson [29] have carried out a detailed study of bifurcation phenomena in plane strain tension for a broad class of incompressible rate independent solids and Young [30] has carried out a similar study for plane strain compression. In a program of monotonically increasing tension, Hill and IIutchinson [29] find that the earliest bifurcation corresponds to the long wavelength diffuse necking mode. For solids described by smooth yield surface plasticity this is, in essence, the only attainable bifurcation mode. However, for a solid described by a corner theory of plasticity, a very short wavelength surface wave mode subsequently becomes available. At larger strains still, a shear band bifurcation occurs.

The onset of diffuse necking depends very much on the geometry of the body. Figures 2 and 3, from Larsson et al. [31], show observed instability modes in long, closed-end aluminum alloy tubes subject to internal pressure.

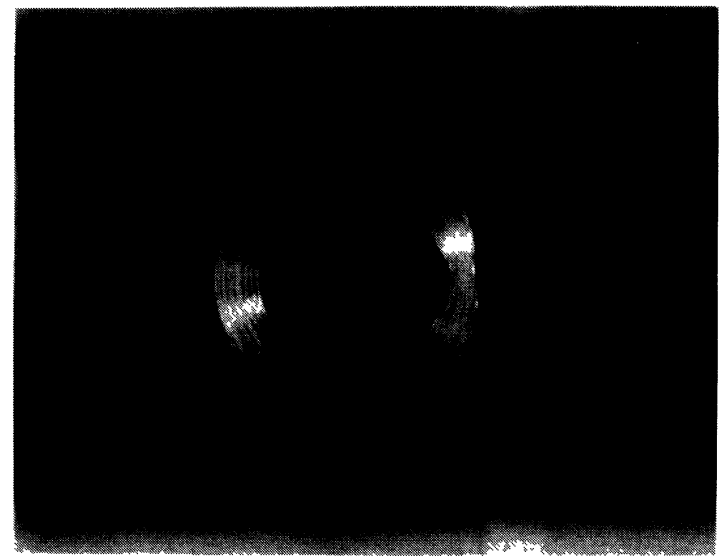

Figune-2 Deformed cross section of an aluminum alloy tube. From [31].

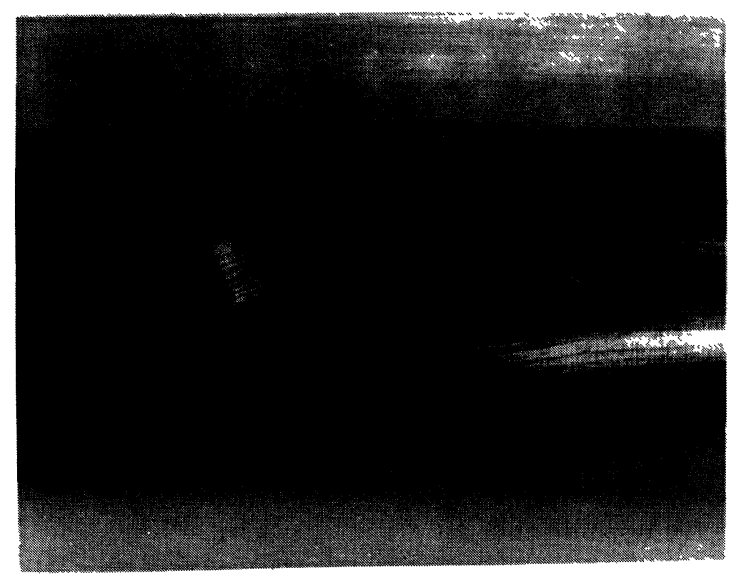

Figune-3 Surface waves on an aluminum alloy tube. From [31]. 
The tubes retain cylindrical symmetry until somewhat beyond the maximum pressure point. Then, a diffuse necking mode develops that involves localized thinning consistent with instability predictions. A very short wavelength surface deformation pattern is observed in the highly strained region. Subsequently, the tube fails in a shear fracture mode. The development of surface waves requires the presence of a free surface and so is a geometric instability in the sense in which that term is used here. However, because of the very short wavelength of this mode, it is not sensitive to the curvature of the body. Hutchinson and Tvergaard [22] have given a very general analysis of such surface instabilities.

\subsection{Material Instabilities}

Here, all-around displacement conditions are imposed so as to rule out geometric instabilities. An element of a solid is considered subject to displacement boundary conditions that in a homogeneous (and homogeneously deformed) solid would give rise to a uniform deformation gradient field. Conditions are sought under which bifurcation into a localized band mode can occur. The basic principles of this analysis are due to Hadamard [32] for elastic solids and Hill [33], Mandel [34], Thomas [35] and Rice [8] for elastic-plastic solids. Within this framework the onset of localization coincides with the loss of ellipticity of the equations governing rate equilibrium. Band type analyses have served as a basis for investigating the role of various constitutive features in promoting localization, see e.g. Rice [8], Needleman and Rice [9] and Hutchinson and Tvergaard [25]. In particular, the important role of vertex effects in the development of localization in strain hardening solids is revealed by such analyses.

Current values of field quantities and material properties inside and outside the band are presumed identical so that one possible solution for the incremental quantities corresponds to the homogeneous one. At the considered stage of the deformation history, suppose that within a thin planar band of orientation $\mathbf{n}$ in the reference configuration incremental field quantities are permitted to take on values differing from the uniform values outside the band. The band is presumed sufficiently narrow to be regarded as homogeneously deformed.

Two requirements must be satisfied across the band interface First, compatibility requires (Hadamard [32], Hill [33], Mandel [34], Thomas [35] and Rice [8]),

$$
\dot{\mathbf{F}}_{b}=\dot{\mathbf{F}}_{o}+\dot{\mathbf{q}} \otimes \mathbf{n}
$$

where ()$_{b}$ denotes field quantities inside the band and ()$_{o}$ denotes corresponding quantities outside the band.

For an incompressible solid, the strain rate jump across the band is a shear strain rate jump and, hence, the band is a shear band.

Next, incremental equilibrium requires

$$
\mathbf{n} \cdot\left(\dot{\mathbf{s}}_{b}-\dot{\mathbf{s}}_{o}\right)=\mathbf{0}
$$

For classical plasticity (3.8), where $\mathbf{K}$ has two branches, one corresponding to plastic loading and the other to elastic unloading, and for a material element subject to continued plastic loading, a localization bifurcation is possible when

$$
\left[\mathbf{n} \cdot \mathbf{K}_{\tan } \cdot \mathbf{n}\right] \cdot \dot{\mathbf{q}}=\mathbf{0}
$$

where the operator .. is defined so that the component form of (4.5) is $n_{i} K_{t a n}^{i j k l} n_{k} \dot{q}_{l}$ and $\mathbf{K}_{t a n}$ corresponds to the plastic loading branch of the tensor of moduli. The onset of localization first becomes possible at the earliest stage in the deformation history at which (4.5) has a nontrivial solution. For a non-trivial solu- tion, the determinant of coefficients in (4.5) must vanish and the governing rate equations lose ellipticity.

For the rate dependent elastic-viscoplastic solids described by (3.9) the plasticity is contained in $\dot{\mathbf{Q}}$. Since $\dot{\mathbf{Q}}$ is independent of incremental quantities it is the same both inside and outside the band. Hence, the counterpart to (4.5) is

$$
\left[\mathbf{n} \cdot \mathbf{K}_{\text {elastic }} \cdot \mathbf{n}\right] \cdot \dot{\mathbf{q}}=0
$$

As long as stress levels remain small compared to elastic stiffnesses, the only solution to (4.6) is the trivial one and a localization bifurcation does not occur. What bears emphasis here is the different mathematical character of the governing equations for rate independent material behavior and for rate dependent material behavior. For the viscoplastic constitutive relation, the mathematical difficulties associated with the loss of ellipticity in the case of rate independent material behavior do not arise. Thus, it is particularly significant that the phenomenology of shear band development can be the same for both rate dependent material behavior and for rate independent material behavior, as illustrated in Peirce et al. [10] and Becker and Needleman [41].

Even when a shear localization bifurcation is precluded at achievable strains, small initial imperfections can induce localization. A general framework for imperfection based localization analyses has been presented by Rice [8], which proceeds in the spirit of Marciniak and Kuczynski's [36] analysis of localized necking in thin sheets. Both (4.3) and (4.4) must still be satisfied. However, when an initial imperfection is present current values of field quantities and material properties inside the band, in general, differ from those outside the band. Using (2.6) in (4.3) and (4.4) gives

$$
\left[\mathbf{n} \cdot \mathbf{K}_{b} \cdot \mathbf{n}\right] \cdot \dot{\mathbf{q}}=\mathbf{n} \cdot\left(\mathbf{K}_{o}-\mathbf{K}_{b}\right): \dot{\mathbf{F}}_{o}+\mathbf{n} \cdot\left(\dot{\mathbf{Q}}_{b}-\dot{\mathbf{Q}}_{o}\right)
$$

where $\dot{\mathbf{F}}_{o}$ is prescribed. At each stage of the deformation history, (4.7) is a set of three equations for the components of $\dot{\mathbf{q}}$. Time integration then gives the histories inside and outside the band. Analyses based on (4.7) are applicable for both rate independent and rate dependent solids. The imperfection analysis is important since, for example, a localization bifurcation is ruled out for rate depedent solids but can occur when very small initial imperfections are present. It also permits the influence of corner characteristics on shear band development to be explored. Hutchinson and Tvergaard [25] have carried out such a study and have found that due to the stiffening associated with a change in loading path, the deformations in a shear band can saturate, which also occurs in the full finite element solutions of Tvergaard et al. [38].

In a band analysis, one homogeneous deformation state is assumed to prevail within the band and a different homogeneous state is presnt outside the band. The more general case, where localization develops from a non-homogeneous deformation state is more complex and requires a numerical computation. However, the role played by various aspects of the material's constitutive description is as revealed by the band analysis. Furthermore, knowing critical shear band angles is useful in designing effective meshes for localization problems, Tvergaard et al. [38].

Figure 4, from Burke and Nix [37], shows the results of a numerical solution for neck development in plane strain tension based on rate independent isotropic hardening plasticity theory. This analysis captures the initiation and growth of the diffuse necking mode but there is no tendency for the deformation pattern to shift to one involving localized shearing as is so commonly observed for structural metals. 


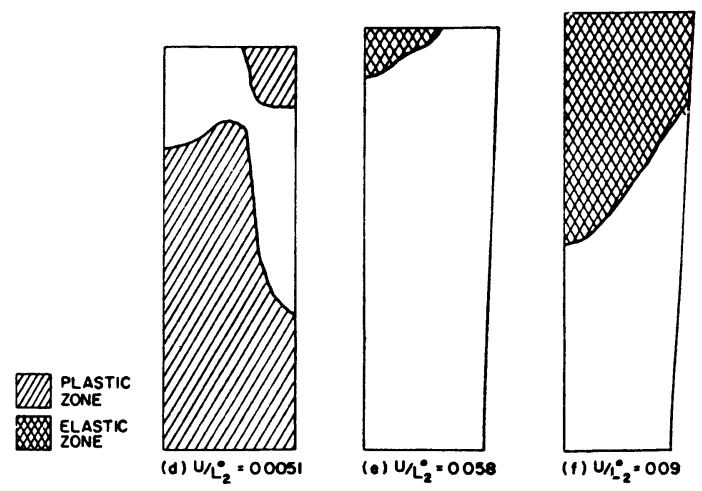

Figune-4 Neck development in a plane strain tensile specimen with the material characterized by isotropic hardening plasticity theory. From [37].
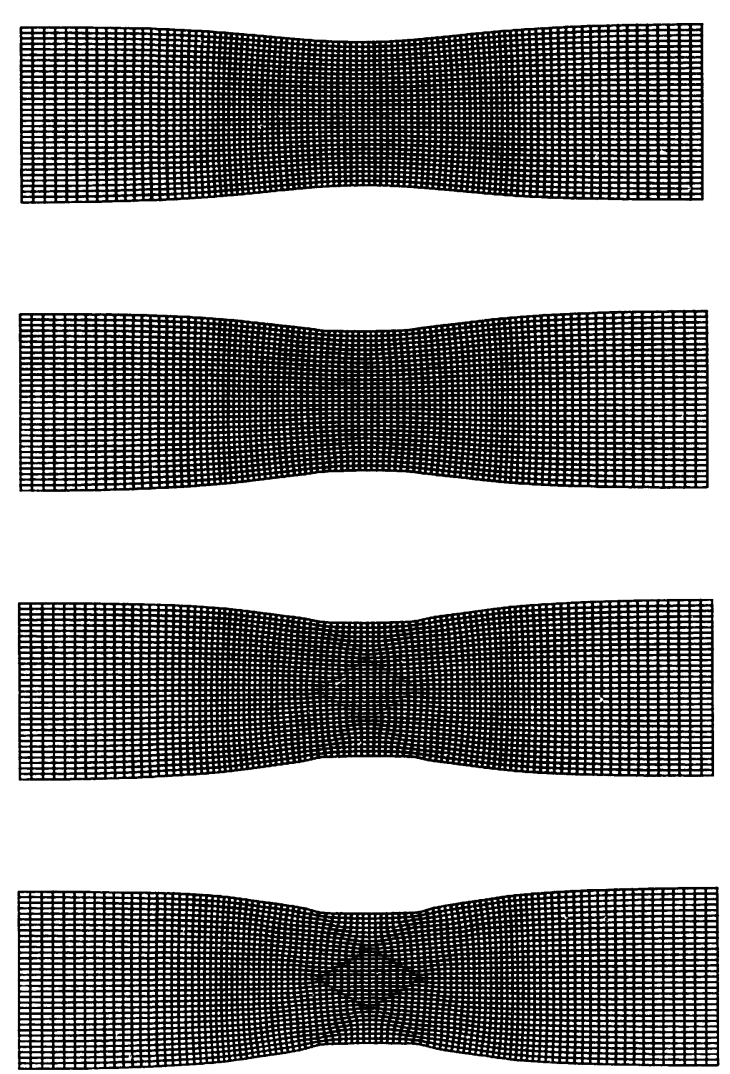

Figune-5 Deformed finite element meshes at various stages of extension in a plane strain tensile specimen. The material is strain hardening and characterized by $J_{2}$ corner theory. From [38].

Figure 5, from Tvergaard et al. [38], shows the course of neck development based on the $J_{2}$ corner theory of Christoffersen and Hutchinson [21]. The actual computations were carried out for one quadrant and symmetry boundary conditions were imposed. Shear bands develop naturally during the course of the calculation. The orientation of the band, or bands, is in good agreement with that predicted from a shear band bifurcation analysis based on (4.5).

\section{Shear Localization in a Temperature Dependent Solid}

LeMonds and Needleman [14] studied the development of localization from a small material inhomogeneity incorporating the effects of thermal softening, strain hardening, strain rate sensitivity and heat conduction. The specific boundary value problem analyzed was plane strain compression of a block of material containing a doubly periodic array of inhomogeneities as shown in Fig. 8. The symmetry permits the boundary value problem for the array to be reduced to a boundary value problem for one square cell. No heat flux is permitted between cells so that the overall response is adiabatic. However, heat conduction is accounted for within each cell. The material properties were chosen to be representative of a 4340 steel studied experimentally by Hartley [39]. In particular, the strain hardening exponent, $N$, was taken as $N=0.08$ and the strain rate sensitivity exponent, $m$, as $m=0.01$.

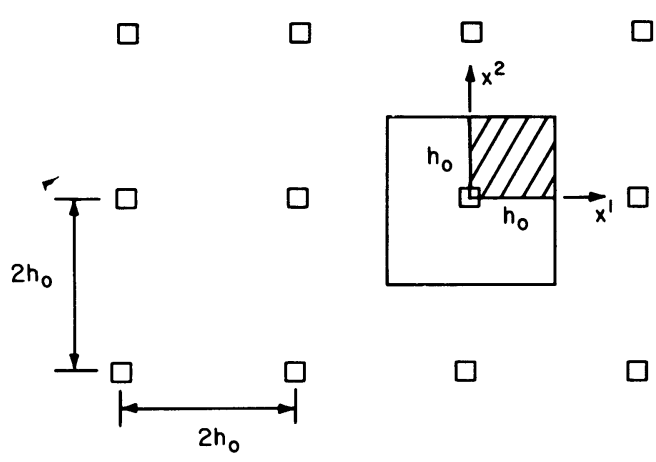

Figure-6 A doubly periodic array of inhomogeneities. The shaded area marks the domain analyzed numerically.

An initial inhomogeneity was prescribed in the form of a soft spot. The soft spot was modelled by specifying a value of the reference strength $\sigma_{0}$ in the quadrilateral element nearest the origin equal to eighty percent of its value in the remaining elements. Three prescribed strain rates were considered $\dot{\epsilon}_{n} / \dot{\epsilon}_{0}=5 \times 10^{5}$, $\dot{\epsilon}_{n} / \dot{\epsilon}_{0}=5 \times 10^{4}$ and $\dot{\epsilon}_{n} / \dot{\epsilon}_{0}=5 \times 10^{3}$. Since $\dot{\epsilon}_{0}=10^{-3} \mathrm{sec}^{-1}$ these correspond to applied strain rates of $\dot{\epsilon}_{n}=500 \mathrm{sec}^{-1}$, $\dot{\epsilon}_{n}=50 \mathrm{sec}^{-1}$ and $\dot{\epsilon}_{n}=5 \mathrm{sec}^{-1}$. The reference temperature $T_{0}$ in (3.13) and (3.16) was taken as $20^{\circ} \mathrm{C}$.

Figures 7 and 8 show the computed effect of heat conduction on the response of an isotropically hardening solid at a prescribed strain rate of $\dot{\epsilon}_{n}=500 \mathrm{sec}^{-1}$. Under adiabatic conditions $(k=0$ in (2.8)), Fig. 7, a sharp shear band forms for the isotropically hardening solid. Heat conduction delays the onset of shearing and broadens the shear band that does develop as seen in Fig. 8 . At lower strain rates, the shear band continues to broaden as depicted in Fig. 9 where $\dot{\epsilon}_{n}=5 \mathrm{sec}^{-1}$ and at $\dot{\epsilon}_{n}=5 \mathrm{sec}^{-1}$ LeMonds and Needleman [14] found that the deformation remained essentially uniform out to a height reduction of $60 \%$.

By way of contrast for a kinematically hardening solid and with heat conduction accounted for, sharp shear bands develop at $\dot{\epsilon}_{n}=500 \mathrm{sec}^{-1}$ as shown in Fig. 10. 


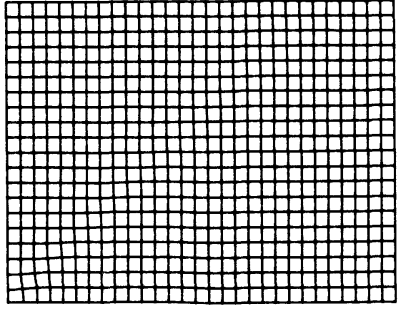

(a)

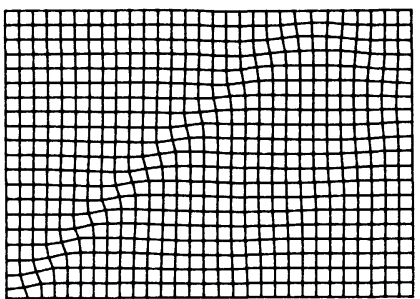

(b)

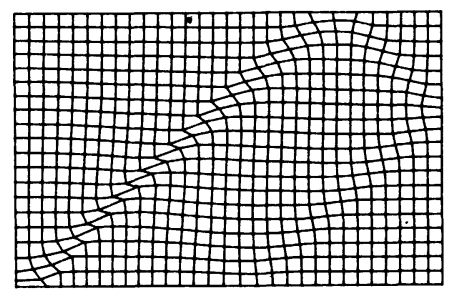

(c)

Frgure-7 Deformed finite element meshes showing shear band development in an isotropically hardening solid under adiabatic conditions and with $\dot{\epsilon}_{n}=500 \mathrm{sec}^{-1}$. From [14].

In fact, even as constant temperature conditions are approached with an imposed strain rate of $\dot{\epsilon}_{n}=5 \sec ^{-1}$ a well defined shear band is evident, Fig. 11.

The behavior shown in Fig. 11 is in accord with the results of Hutchinson and Tvergaard's [25] study of plane strain shear band formation in rate independent solids deforming isothermally. The increased curvature of the flow potential surface of the kinematically hardening solid acts as a "rounded" vertex and permits localization at physically achievable strain levels.

LeMonds and Needleman's [14] results illustrate the significance of the multi-axial constitutive characterization of the material even in the presence of thermal softening. For the kinematic hardening solid, thermal softening promotes localization in that localized straining occurs sooner and more sharply when thermal softening effects are significant. But localization still is found when conditions of nearly uniform temperature prevail. On the other hand, for the isotropic hardening material model, localization requires strong thermal softening.

\section{Discussion}

The aim here has been to illustrate the significance for plastic instability phenomena of the material's three dimensional constitutive description, even in circumstances where a softening mechanism plays a major role in precipitating the instability. The specific example involved thermal softening, but similar effects have been noted in studies where the softening mechanism is micro-void nucleation and growth, Mear and Hutchinson [40], Becker and Needleman [41] and Tvergaard [42].

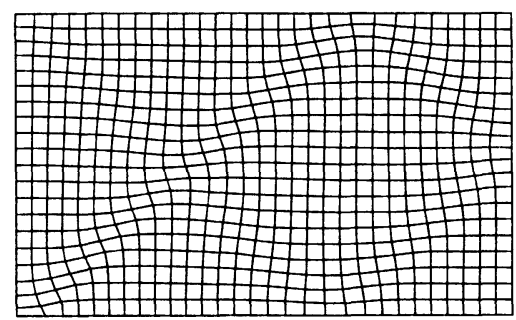

(a)

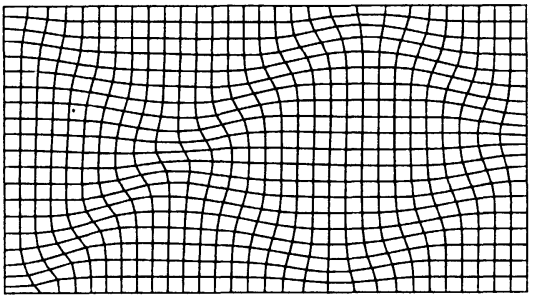

(b)

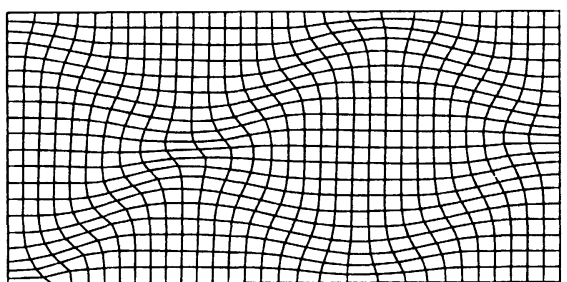

(c)

Figure-8 Deformed finite element meshes showing shear band development in an isotropically hardening solid with heat conduction accounted for and with $\dot{\epsilon}_{n}=500 \mathrm{sec}^{-1}$. From [14].
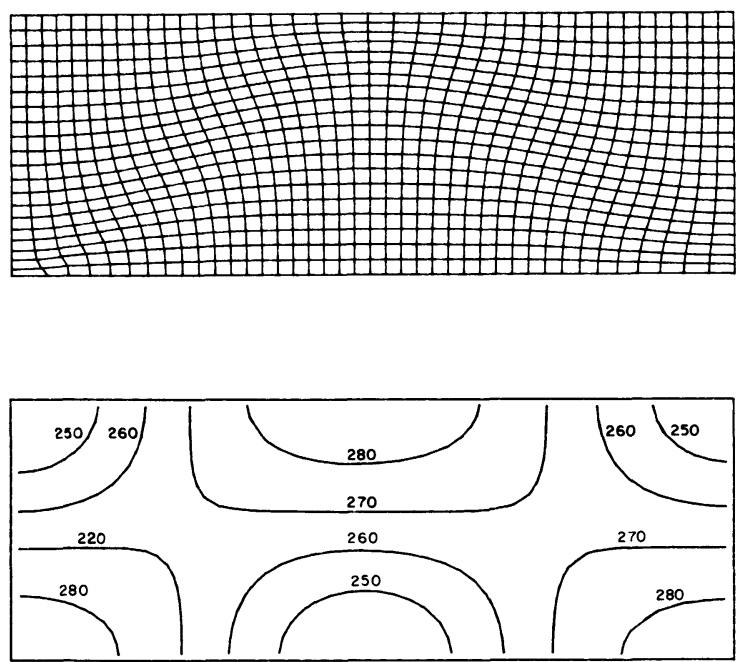

Frgure-9 Results at one stage of compression for an isotropically hardening solid an isotropically hardening solid with heat conduction accounted for and with $\dot{\epsilon}_{n}=50 \mathrm{sec}^{-1}$; the top figure shows the deformed finite element mesh and the bottom figure shows contours of constant temperature in degrees Celsius. From [14]. 


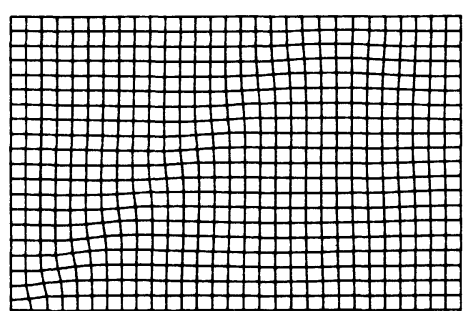

(a)

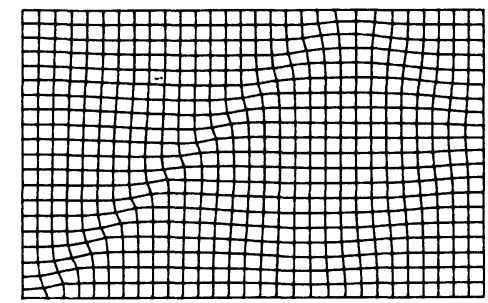

(b)

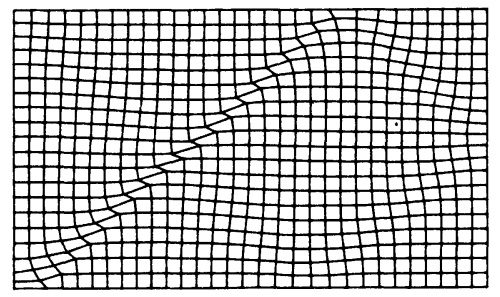

(c)

Figure-10 Deformed finite element meshes showing shear band development in an kinematically hardening solid with heat conduction accounted for and with $\dot{\epsilon}_{n}=500 \mathrm{sec}^{-1}$. From [14].

The incorporation of heat conduction explicitly introduces a length scale into the analysis, where the characteristic length is $\left(k / \rho c_{p} \dot{\epsilon}_{n}\right)^{1 / 2}$, specifying a length over which heat conduction effects are significant. Additionally, material rate dependence implicitly introduces a length scale into the boundary value problem. This length scale is one characterizing the imperfection or inhomogeneity, as discussed by Needleman [46].

Hence, in the analyses of LeMonds and Needleman [14] there are two characteristic lengths; one associated with the inhomogeneity and the other with heat conduction. The issue of characteristic length scales arises in analyses of localization because for a rate independent solid deforming isothermally and developing a shear band there is no characteristic length to set the shear band width. In numerical calculations a length scale is introduced via the discretization. As a consequence numerical solutions to localization problems for rate independent solids exhibit an inherent mesh dependence and global quantities, such as the overall stiffness characteristics of the body, depend on the mesh size used to resolve the band of localized deformations. This has been observed in a variety of contexts and discussed from diverse perspectives, e.g. Tvergaard et al. [38], Pietruszcak and Mroz [43]: Tvergaard [44], Belytschko, Bazant, Hyun and Chang [45] and Needleman [46].

When material rate dependence is accounted for, there is no loss of ellipticity in quasi-static problems as long as stress levels remain small compared to elastic stiffnesses. Even though the mathematical character of the governing rate equations funda-

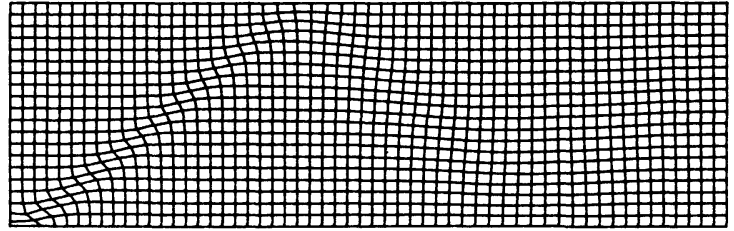

(a)

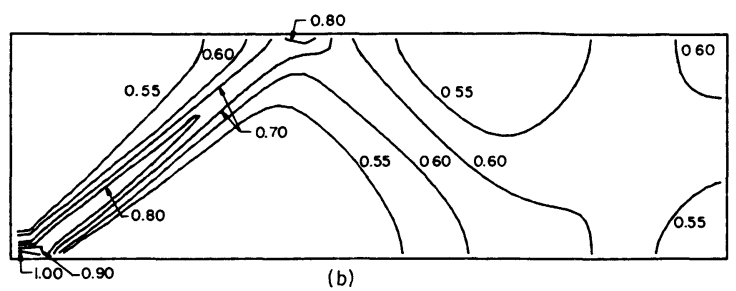

(b)

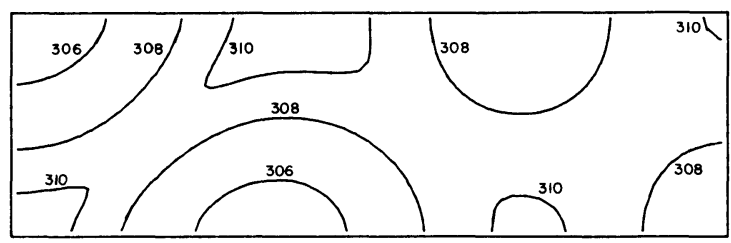

(c)

Figure-11 Results at one stage of compression for a kinematically hardening solid with $\dot{\epsilon}_{n}=5 \mathrm{sec}^{-1}$. (a) Deformed finite element mesh. (b) Contours of constant maximum principle logarithmic strain. (c) Contours of constant temperature in degrees Celsius.

mentally differ, the phenomenology of localization can be the same for both rate dependent and rate independent material behavior e.g., Peirce et al. [10] and Becker and Needleman [41]. However, since the governing equations for rate dependent solids remain elliptic, the sort of pathological mesh dependence encountered for rate independent solids appears to be precluded.

There are computational issues associated with localization that arise whether the material is characterized as rate independent or rate dependent. For example, in any conventional finite element calculation the mesh size sets the minimum width of a shear band. Hence, if there is a characteristic material length that sets the shear band width, mesh resolution must be fine enough to resolve this width. There does not now appear to be a usable way to assess whether or not, in a specific problem, a given mesh does possess such resolution. Furthermore, the ability of a finite element mesh to resolve localized shearing at angles oblique to the element boundaries significantly affects the predicted course of shear band development, as discussed by Tvergaard et al. [38].

Of course, in any particular circumstance, there is the question as to what the appropriate characteristic length scale is. In certain circumstances, the dimensions of some microstructural feature, e.g. a grain size, may set the length scale. In fact, any reference to homogeneous plastic flow in metals deforming by dislocation slip appears to imply some size scale below which inhomogenieties in deformation patterns are ignored, since on the discrete dislocation scale plastic flow is inherently nonuniform. Physically appropriate and mathematically tractable ways of incorporating length scales into continuum descriptions of localization problems merit further exploration. 


\section{Acknowledgement}

The support of the U.S. National Science Foundation (Solid Mechanics Program) through grant MSM8618007 is gratefully acknowledged.

\section{References}

[1] R.J. Asaro, Acta Metall. 27, (1979) 445.

[2] I.L. Dillamore, J.G. Roberts and A.C. Bush, J. Met. Sci. 13, (1979) 73.

[3] Y.W. Chang and R.J. Asaro, Acta Metall. 29 (1981) 241.

[4] K. Morii, M. Mera and Y. Nakayama, Trans. Japan Inst. Met. 18, (1977) 7.

[5] C. Zener and J.H. Hollomon, J. Appl. Phys. 15 (1944) 22.

[6] H.C. Rogers, "Adiabatic Shearing-A Review," Drexel University Report (1974).

[7] L.S. Costin, E.E. Crisman, R.H. Hawley and J. Duffy in "Proceedings Second Conference on Material Properties at High Rates of Strain," edited by J. Harding, 90, The Institute of Physics, Bristol and London, Conference Series No. 17, (1979).

[8] J.R. Rice in "Theoretical and Applied Mechanics," Proc. 14th Int. Congr. Theoret. Appl. Mech., edited by W.T. Koiter, 207, North Holland, Amsterdam, (1977).

[9] A. Needleman and J.R. Rice in "Mechanics of Sheet Metal Forming," edited by D.P. Koistinen and N.-M. Wang, 237, Plenum, New York, (1978).

[10] D. Peirce, R.J. Asaro and A. Needleman, Acta Metall. 31, (1983) 1951.

[11] A. Needleman and V. Tvergaard in "Finite Elements-Special Problems in Solid Mechanics Vol. 5," edited by J.T. Oden and G.F. Carey, 94, Prentice-Hall, Englewood Cliffs, (1984).

[12] R.J. Asaro, Adv. Appl. Mech. 23 (1983) 1.

[13] V. Tvergaard in "Constitutive Equations: Macro and Computational Aspects," edited by K.J. Willam, 179, ASME, New York (1984).

[14] J. LeMonds and A. Needleman, Mech. Matl. 5 (1986) 339.

[15] G.I. Taylor and H. Quinney, Proc. Roy. Soc. London A143 (1934) 307.

[16] R. Hill, J. Mech. Phys. Solids 15 (1967) 79.

[17] J.W. Hutchinson, Proc. Roy. Soc. London A318 (1970) 247.

[18] S.S. Hecker in "Constitutive Equations in Viscoplasticity," ASME AMD 20 (1976) 1.

[19] J. Pan and J.R. Rice, Int. J. Solids Struct. 19 (1983) 973.

[20] R.J. Asaro and A. Needleman, Acta Metall. 33 (1985) 923.

[21] J. Christoffersen and J.W. Hutchinson, J. Mech. Phys Solids 27 (1979) 465.
[22] J.W. Hutchinson and V. Tvergaard, Int. J. Mech. Sci. 22 (1980) 339.

[23] J.R. Rice, J. Appl. Mech. 92 (1970) 728.

[24] V. Tvergaard, Int. J. Mech. Sci. 20 (1978) 651.

[25] J.W. Hutchinson and V. Tvergaard, Int. J. Solids Struct. 17 (1981) 451.

[26] R. Hill, J. Mech. Phys. Solids 6 (1958) 236.

[27] R. Hill Adv. Appl. Mech. 18 (1978) 1.

[28] B. Raneicki and O. Bruhns, J. Mech. Phys. Solids 29 (1981) 153.

[29] R. Hill and J.W. Hutchinson, J. Mech. Phys. Solids 23 (1975) 239.

[30] N.Y.B. Young, J. Mech. Phys. Solids 24 (1976) 77.

[31] M. Larsson, A. Needleman, V. Tvergaard and B. Storåkers, J. Mech. Phys. Solids 30 (1982) 121.

[32] J.J. Hadamard, "Leçons sur la Propagation des Ondes et les Équations de L'Hydrodynamique," Libraire Scientifique A. Hermann, Paris (1903).

[33] R. Hill, J. Mech. Phys. Solids 10 (1962) 1.

[34] J. Mandel, in "Rheology and Soil Mechanics," edited by J. Krautchenko and P.M. Sirieys, 58, Springer-Verlag, Berlin (1966).

[35] T.Y. Thomas, "Plastic Flow and Fracture in Solids," Academic Press, New York (1961).

[36] Z. Marciniak and K. Kuczynski, Int. J. Mech. Sci. 9 (1967) 609.

[37] M.A. Burke and W.D. Nix, Int. J. Solids Struct. 15 (1979) 379.

[38] V. Tvergaard, A. Needleman and K.K. Lo, J. Mech. Phys. Solids 29 (1981) 115.

[39] K.A. Hartley, "Temperature Measurements During the Formation of Shear Bands at High Rates of Deformation," Ph. D. Thesis, Brown University (1986).

[40] M.E. Mear and J.W. Hutchinson, Mech. Matl. 4 (1985) 395.

[41] R. Becker and A. Needleman, J. Appl. Mech. 108 (1986) 491.

[42] V. Tvergaard, J. Mech. Phys. Solids 35 (1987) 43.

[43] S.T. Pietruszcak and Z. Mroz, Int. J. Num. Meth. Engr. 10 (1981) 327.

[44] V. Tvergaard, J. Mech. Phys. Solids 30 (1982) 399.

[45] T. Belytschko, Z.P. Bazant, Y.-W. Hyun and T.-P. Chang, Comp. Struct. 23 (1986) 163.

[46] A. Needleman, "Material Rate Dependence and Mesh Sensitivity in Localization Problems," Brown University Report ONR-N00014-86-K-0235/1 (1987). 